\title{
A mutation in a functional Sp I binding site of the telomerase RNA gene (hTERC) promoter in a patient with Paroxysmal Nocturnal Haemoglobinuria
}

W Nicol Keith1, Tom Vulliamy2, Jiangqin Zhao1, Cem Ar ${ }^{3}$, Can Erzik ${ }^{4}$, Alan Bilsland ${ }^{1}$, Birsen Ulku ${ }^{3}$, Anna Marrone ${ }^{2}$, Philip J Mason*2,5, Monica Bessler ${ }^{5}$, Nedime Serakinci ${ }^{6}$ and Inderjeet Dokal ${ }^{2}$

Address: ${ }^{1}$ Cancer Research UK, Department of Medical Oncology, Cancer Research UK Beatson Laboratories, University of Glasgow, Garscube Estate, Switchback Road, Glasgow G61 1BD, UK, ${ }^{2}$ Department of Haematology-Division of Investigative Science, Imperial College London, Hammersmith Hospital, London, UK, ${ }^{3}$ Department of Haematology, Cerrahpasa Medical Faculty, Istanbul University, Turkey, ${ }^{4}$ Medical Faculty, Marmara University, Turkey, ${ }^{5}$ Department of Internal Medicine, Division of Hematology, Washington University School of Medicine, St.Louis, MO, 63110, USA and 'Department of Human Genetics, Bartholin Building, University of Aarhus, Universitetsparken, 8000 Aarhus C, Denmark

Email: W Nicol Keith - n.keith@beatson.gla.ac.uk; Tom Vulliamy - t.vulliamy@imperial.ac.uk; Jiangqin Zhao - J.Zhao@beatson.gla.ac.uk; Cem Ar - muhcar@superonline.com; Can Erzik - ursusc@superonline.com; Alan Bilsland - a.bilsland@beatson.gla.ac.uk; Birsen Ulku - ubulku@superonline.com; Anna Marrone - a.marrone@imperial.ac.uk; Philip J Mason* - Pmason@im.wustl.edu; Monica Bessler - mbessler@im.wustl.edu; Nedime Serakinci - nedi@humgen.au.dk; Inderjeet Dokal - i.dokal@imperial.ac.uk

* Corresponding author

Published: 22 June 2004

BMC Blood Disorders 2004, 4:3
Received: 15 March 2004

Accepted: 22 June 2004

This article is available from: http://www.biomedcentral.com/l47I-2326/4/3

(C) 2004 Keith et al; licensee BioMed Central Ltd. This is an Open Access article: verbatim copying and redistribution of this article are permitted in all media for any purpose, provided this notice is preserved along with the article's original URL.

\begin{abstract}
Background: Mutations in the gene coding for the RNA component of telomerase, hTERC, have been found in autosomal dominant dyskeratosis congenita (DC) and aplastic anemia. Paroxysmal nocturnal hemoglobinuria $(\mathrm{PNH})$ is a clonal blood disorder associated with aplastic anemia and characterized by the presence of one or more clones of blood cells lacking glycosylphosphatidylinositol (GPI) anchored proteins due to a somatic mutation in the PIGA gene.
\end{abstract}

Methods: We searched for mutations in DNA extracted from $\mathrm{PNH}$ patients by amplification of the $h T E R C$ gene and denaturing high performance liquid chromatography (dHPLC). After a mutation was found in a potential transcription factor binding site in one patient electrophoretic mobility shift assays were used to detect binding of transcription factors to that site. The effect of the mutation on the function of the promoter was tested by transient transfection constructs in which the promoter is used to drive a reporter gene.

Results: Here we report the finding of a novel promoter mutation $(-99 \mathrm{C}->\mathrm{G})$ in the $h T E R C$ gene in a patient with PNH. The mutation disrupts an SpI binding site and destroys its ability to bind SpI. Transient transfection assays show that mutations in this $h T E R C$ site including C-99G cause either up- or downregulation of promoter activity and suggest that the site regulates core promoter activity in a context dependent manner in cancer cells.

Conclusions: These data are the first report of an $h T E R C$ promoter mutation from a patient sample which can modulate core promoter activity in vitro, raising the possibility that the mutation may affect the transcription of the gene in hematopoietic stem cells in vivo, and that dysregulation of telomerase may play a role in the development of bone marrow failure and the evolution of $\mathrm{PNH}$ clones. 


\section{Background}

Dyskeratosis congenita is an inherited bone marrow failure syndrome whose characteristic features are nail dystrophy, abnormal skin pigmentation and mucosal leucoplakia [1]. There are X-linked, autosomal dominant and recessive forms of the disease. The X-linked form is due to mutations in the gene, $D K C 1$, which codes for the nucleolar protein dyskerin [2]. Dyskerin is a component of snoRNP complexes involved in rRNA maturation [3] and is also associated with the telomerase complex [4]. Autosomal dominant DC is due to mutations in the gene, hTERC [5] encoding the telomerase RNA component, suggesting the pathology of DC is largely due to defective telomerase. The recent finding of hTERC mutations in cases presenting with aplastic anaemia [6,7] has introduced the possibility that defects in telomerase may be more widespread than previously thought.

Paroxysmal nocturnal haemoglobinuria (PNH) is a clonal blood disorder which often develops in association with aplastic anaemia [8]. In PNH a somatic mutation in the PIGA gene disrupts the synthesis of GPI anchors resulting in the growth of one or more clones of GPI- blood cells (which lack GPI anchored proteins such as CD55 and CD59) [9]. It is presumed that these cells survive in the aplastic marrow because they evade the insult that is killing the other cells (GPI+) and leading to aplasia [8]. The nature of the insult is not known but it is presumed to involve one or more GPI linked proteins. PNH is not known to be inherited. While there are cases of familial or constitutional AA the vast majority of cases are idiopathic. However the observation that idiopathic AA often occurs after exposure to a drug or other chemical that many people are exposed to suggests that there may be a genetic predisposition to the development of AA. Here we present a case of $\mathrm{PNH}$ in which the proband was found to have a mutation in an Sp1 binding site in the promoter of hTERC. In vitro analysis of the mutated promoter suggests the mutation may affect $h T E R C$ promoter activity and thus might influence telomerase levels.

\section{Methods \\ Case History}

An 18 year-old Turkish man presented with a three month history of fatigue, nose and gum bleeding. He was found to have pancytopenia at his local Hospital and was referred to the Haematology Department of the Cerrahpasa Medical Faculty (University of Istanbul) in August 2001 for further management. His past medical history was unremarkable and there was no recent exposure to chemicals or drugs. Physical examination was unremarkable except for marked pallor and petechiae. Spleen was not palpable. His initial investigations were as follows: Hb $4.1 \mathrm{~g} / \mathrm{dl}$, WBC $1.4 \times 10^{9} / 1\left(0.6 \times 10^{9} / 1\right.$ neutrophils $)$, platelets $10.0 \times 10^{\%} / \mathrm{l}$, reticulocytes $0.4 \%$, MCV $115.4 \mathrm{fl}$.
Blood film showed anisocytosis with prominent poikilocytes and polychromasia. The serum iron, iron binding capacity, vitamin B12, folic acid and haptoglobin measurements were within the normal range. His $\mathrm{LDH}$ and indirect bilirubin were slightly elevated.

Bone marrow biopsy revealed variable cellularity between $<5 \%$ to $60 \%$. Cellular parts of the marrow displayed focal erythroid hyperplasia with megaloblastoid and dyserythropoietic features. Myelopoiesis was relatively reduced and some megakaryocytes were present.

Direct Coombs test was negative. Hemosiderinuria was detected. Flow cytometric analysis revealed absence of CD55 and CD59 on all granulocytes consistent with a diagnosis of PNH. He was initially treated with prednisolone $(1 \mathrm{mg} / \mathrm{kg} / \mathrm{day})$. A moderate increase in the $\mathrm{Hb}$ level and reticulocyte counts (4.6\%) was noted lasting a few weeks. No improvement was seen in the leucocyte and platelet counts. In November 2001 his blood count was as follows: Hb $4.7 \mathrm{~g} / \mathrm{dl}$, WBC $2.4 \times 10^{9} / \mathrm{l}$, platelets $17.0 \times 10^{9} / \mathrm{l}$. He was cushingoid and developed steroid induced myopathy. The prednisolone was discontinued and he was supported with erythrocyte and platelet transfusions. His blood counts were January 2002 (Hb: $4.5 \mathrm{~g} /$ dl, WBC $2.5 \times 10^{9} / 1$, platelets $\left.60 \times 10^{9} / 1\right)$, December 2003 $(\mathrm{Hb}: 5.5 \mathrm{~g} / \mathrm{dl}, \mathrm{WBC} 2.9 \times 10 \% / \mathrm{l}$, Hct: $16 \%$, platelets $13 \times$ $10 \% / 1)$. In July 2002 an archived DNA sample was sent to the Department of Heamatology, Hammersmith Hospital to screen for mutations in the hTERC gene. In December 2003 DNA extracted from a buccal smear was screened for hTERC mutations.

\section{Mutation analysis}

A 654 bp fragment encompassing the hTERC gene was amplified by PCR with the primer pair 5' TCATGGCCGGAAATGGAACT3' and 5'GGGTGACGGATGCGCACGAT3' using the conditions described previously [5]. Fragments were screened for mutation by denaturing high performance liquid chromatography (dHPLC) using the Transgenomic WAVE DNA fragment analysis system. When an abnormal pattern of elution was observed, the sample was re-amplified and subjected to direct sequence analysis by BigDye chain termination cycle sequencing. The products of the reaction were run on a 3700 DNA analyser and the sequences read using the EditView automated DNA sequence viewer (Applied Biosystems Inc). Restriction enzyme digestion of PCR products was carried out according to manufacturers instructions (New England Biolabs).

\section{Construction of reporter plasmids and mutagenesis}

Primers carrying restriction sites were used for PCR with hProm 867 as the template to generate a series of 5' and 3' terminal deletions with compatible ends for cloning as 
Xho I/Hind III fragments into the multiple cloning region of the promoter-less luciferase vector pGL3-basic (Promega, Madison, WI). A two step cloning strategy was used for site-directed mutagenesis to prevent unexpected mutations in luciferase reporter vectors; (i) an hTR 176 bp fragment (2923 wt, spanning from -107 to +69 bp) was cloned into the Xho I/Hind III sites in pCR-Script ${ }^{\mathrm{TM}}$ plasmid vector (Stratagene, La, Jolla CA), which was used as template for PCR using a QuikChange ${ }^{\mathrm{TM}}$ site-directed mutagenesis kit (Stratagene, La, Jolla CA) following the manufacturer's instructions. (ii) All mutation fragments were reconstructed into the Xho I/Hind III sites of pGL3basic vectors and verified by DNA sequencing. Primers carrying the mutations and a second set of primers for subcloning were designed. The PCR reaction was performed with these primers and 2923 wt or 2925 wt as template to create single-site mutation constructs. The double-site mutation construct, $\mathrm{mSp} 1(2)$, harbouring a C-101A/C-100A double substitution, or No-Sp1, harbouring both the C-101A/C-100A double substitution and an ATGT substitution in site Sp1.1 were generated in several separate PCR reactions using mSp1.2 constructs as template. Minimal promoter mutation constructs based on 2925 wt contained the PNH C-99G mutation in site Sp1.2. The following oligonucleotides were used as PCR primers:

hTR23(+69 to +46) Hind III, 5'-cgcaagcttTACGCCCTTCTCAGTTAGGGTTAG-3';

hTR25(+10 to -12) Hind III, 5'-cgcaagctTCCGCAACCCGGTGCGCTGCCG-3';

hTR29(-107 to -88) Xho I, 5-gcgctcgAGCCCGCCCGAGAGAGTGAC-3';

hTR29Fm(-107 to -88) Xho I, 5'-gcgctcgAGCCCGCCGGAGAGAGTGAC-3';

mSp1.1 5'-CGGCGGCCATAGCCTTTATAAGCCGACT-3;

Nucleotides corresponding to promoter sequences are given in uppercase letters from 5 ' end to 3 ' end. Lowercase letters indicate clamps for introduction of restriction enzyme sites. Bold indicates mutation.

\section{Transfection and dual-luciferase reporter assay}

$3.0 \mu \mathrm{g}$ of $h$ TERC promoter plasmids containing firefly luciferase reporters were cotransfected into 5637 bladder cancer cells with an internal Renilla luciferase control, pRL-SV40 (Promega) using Superfect Transfection Reagent (Qiagen) as previously described [10,11]. The total amount of DNA was kept constant at $10 \mu \mathrm{g}$ with Salmon sperm DNA. The activity of both firefly and Renilla luciferase was determined $48 \mathrm{~h}$ later using the Dual Luciferase
Assay kit (Promega). A minimum of three independent transfections were performed in duplicate and specific hTERC promoter activity was normalized to protein as described elsewhere [10-12].

\section{Electrophoretic mobility shift assays (EMSAs)}

Nuclear extracts from cultured 5637 cells were made according to our previous study [10]. EMSAs were performed using the EMSA kit (Promega, E3300). $5.0 \mu \mathrm{g}$ of nuclear extract proteins were incubated in $15 \mu \mathrm{l}$ of reaction containing $4 \%$ glycerol, $1 \mathrm{mM} \mathrm{MgCl}_{2}, 0.5 \mathrm{mM}$ dithiothreitol (DTT), $0.5 \mathrm{mM}$ EDTA, $50 \mathrm{mM} \mathrm{NaCl}, 10 \mathrm{mM}$ Tris$\mathrm{HCl}, \mathrm{pH} 7.5$ and $2.0 \mu \mathrm{g}$ poly(dI-dC) with or without 100 fold molar excess of unlabeled DNA competitors on ice for $15 \mathrm{~min}$, followed by addition of the radiolabelled probe. All DNA-protein complexes were resolved by electrophoresis on $5 \%$ native polyacrylamide.

\section{Results}

In a screen of aplastic anemia samples for hTERC mutations we found a changed dHPLC elution pattern in a Turkish PNH patient. We subsequently analysed 12 other PNH patients in our collection and all appeared normal on dHPLC. DNA sequence analysis showed that the abnormal sample was heterozygous for a C-> G substitution, 99 bp upstream of the hTERC transcript (Figure 1). Since this mutation results in the loss of an AvaI site present in the normal hTERC gene, we were able to confirm the presence of both mutant and normal alleles in this sample by restriction enzyme digestion of the appropriate PCR product (data not shown). This mutation was detected by dHPLC analysis in DNA extracted from a buccal smear taken from the patient, indicating the mutation is constitutional. It was not detected by denaturing HPLC analysis in 100 ethnically matched DNA samples. The C99 residue is located in a core binding sequence for the Sp1 transcription factor (site Sp1.2 [11]). The mutation changes this sequence from 5'CCGCCC3' to 5'CCGCCG3'. We postulated that the mutation might disrupt the Sp1 binding to the hTERC promoter. Initial analysis of transcription factor binding sites in the mutant sequence using the TRANSFAC search tool revealed that the predicted Sp1.2 binding site was lost in the patient mutant sequence.

In order to determine whether the patient C-99G mutation functionally affects Sp1 binding to the Sp1.2 site, we first examined the ability of the mutant sequence to bind Sp1/Sp3 in electromobility shift assays (EMSA). We previously demonstrated by supershift analysis that Sp1/Sp3 bind double stranded oligonucleotides corresponding to the Sp1.2 site with low affinity in vitro but do not bind an oligo in which C-101/C-100 are replaced with AA [13] (Figure 2, h41 m). We now constructed oligos in which C99 was replaced with A (h43 m) or with the G substitution 
Normal
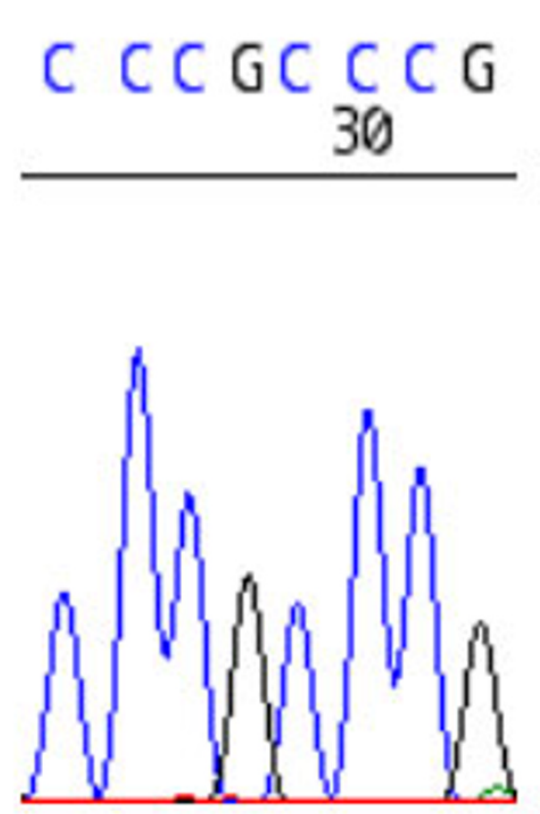

Patient
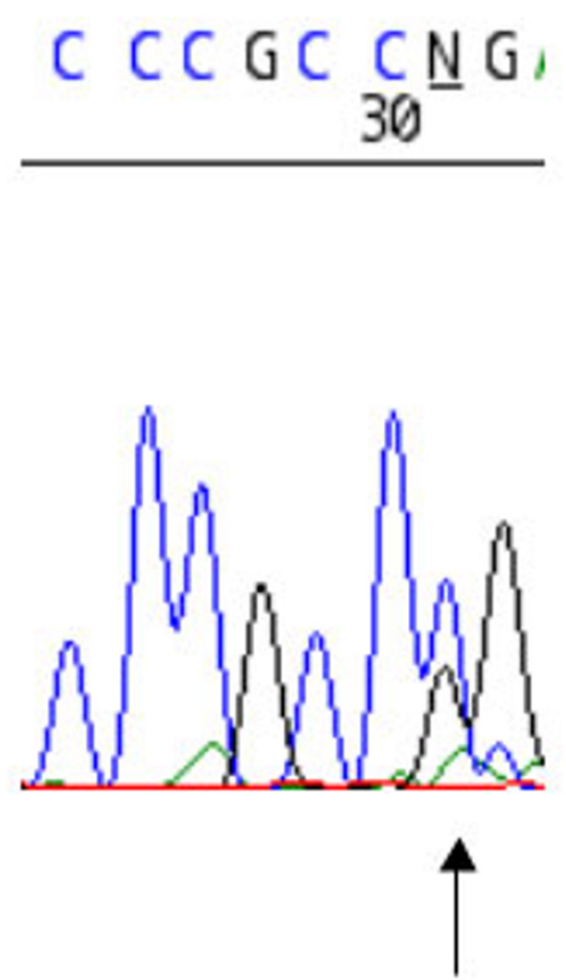

\section{Figure I}

Sequence of $\mathbf{h T E R C}$ in the patient. Sequence traces show that the patient is heterozygous for a C->G substitution (arrowed) upstream of the hTERC gene.

found in the patient (h42 m). Excess of either cold A-99 or patient G-99 mutant failed to compete with the wild type $\mathrm{Sp} 1.2$ oligo for Sp1/Sp3 binding, indicating that an hTERC promoter mutation identified in the PNH patient disrupts the ability of $\mathrm{Sp} 1 / \mathrm{Sp} 3$ to bind a regulatory site in the core promoter. To confirm disruption of Sp1/Sp3 binding, labelled h42 m and h43 m oligos were incubated with 5637 cell nuclear extract, but did not form retarded complexes although complex formation was seen with the wild type Sp1.2 oligo (data not shown).

To investigate whether the loss of Sp1/Sp3 binding might have functional effects on the activity of the hTERC core promoter we constructed wild type and mutant hTERCluciferase reporter plasmids and analysed relative luciferase activity by transient transfection in 5637 bladder cancer cells. We previously showed that the activity of the core hTERC promoter is regulated by $\mathrm{Sp} 1$ sites surrounding the CCAAT box (Sp1.1 and Sp1.2) and by Sp1 sites downstream of the transcriptional start site which have a general repressive role (Sp1.3 and Sp1.4). Removal of the downstream sequence encompassing nucleotides +11 to +69 leads to an approximately 2.5 -fold induction in core promoter activity $[11,13]$. We now examined the effects of the patient C-99G mutation on 

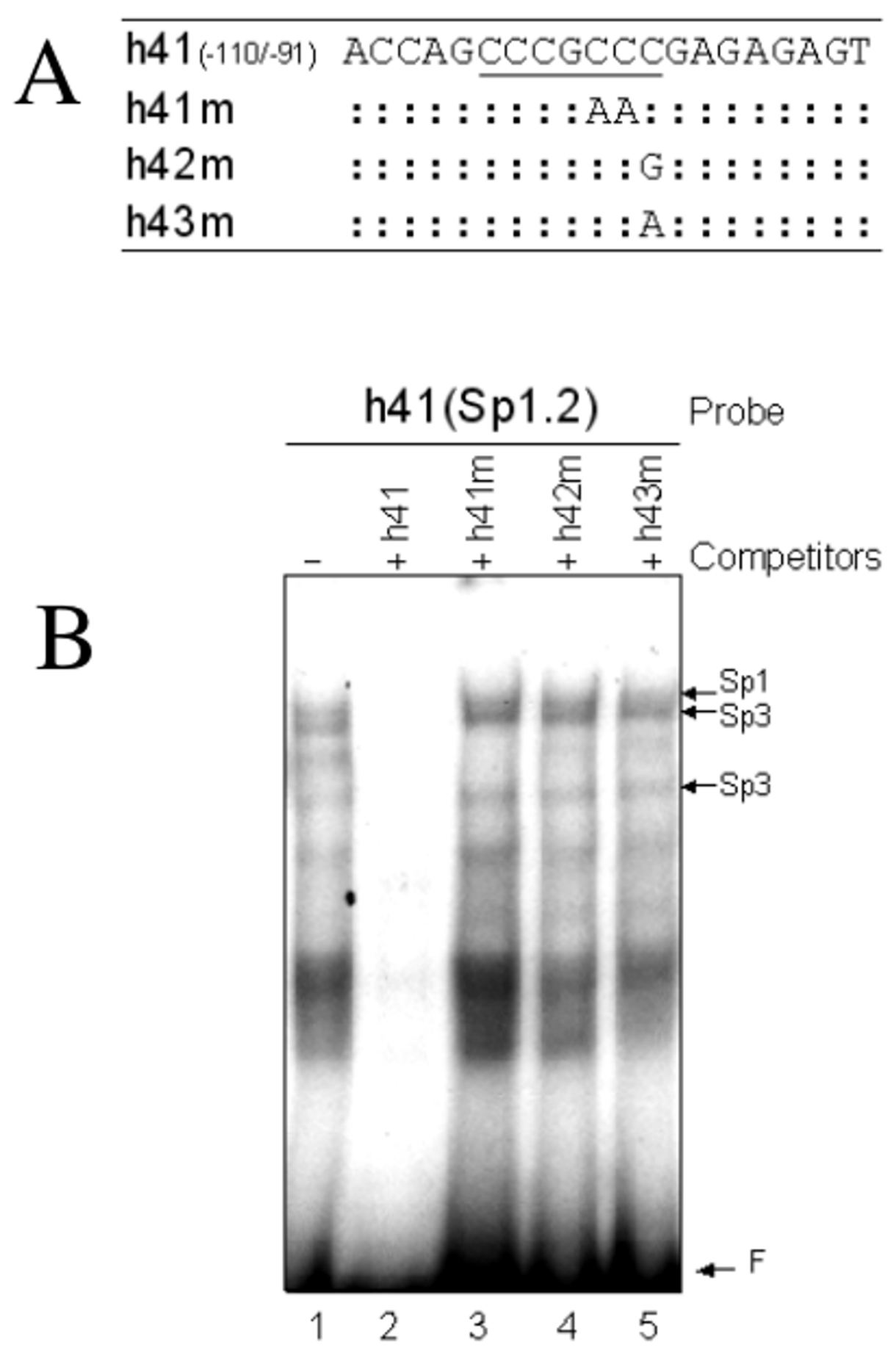

Figure 2

Band shift analysis. A. Oligonucleotides used in EMSA and mutagenesis studies of the $\mathrm{hTERC}$ promoter. The sequence of the wild type oligonucleotides covering identified transcription factor Spl.2 binding sites are shown as h4l. The colon symbol represents bases identical to those in the top row, the mutant sequences are shown underneath. B. Single mutation in SpI.2 site inhibits the SpI binding activity. 5637 nuclear extract was mixed with radiolabelled oligonucleotide probe and analysed by EMSA. Specific DNA/protein complexes are indicated by the arrow on the right hand side. The oligonucleotide h4l was used as a probe and the oligonucleotides used as competitors are indicated at the top oflanes $2-5$. 
hTERC promoter activity in the absence of downstream negative regulation using this minimal construct comprising Sp1.2, CCAAT and Sp1.1 (nucleotides -107 to +10 ).

Consistent with our previous analysis, mutation of the Sp1.1 site led to strong induction of core promoter activity, confirming a repressive role for this site (figure $3 \mathrm{C}$ ). In the minimal promoter context a single mutant construct harbouring the Sp1.2 C-99G mutation had an increased reporter activity, suggesting that this site acts as a repressor of transcription. Additionally, the combined ablation of both Sp1.2 by the PNH C-99G substitution and Sp1.1 led to a weak further increase in promoter activity relative to the Sp1.1 single mutant. Interestingly, we previously observed that inactivation of Sp1/Sp3 binding to Sp1.2 in the context of the larger core promoter $(-107$ to +69$)$ by a C-101A/C-100A double substitution (mSp1.2, figure 3B) results in a slight decrease of hTERC promoter activity, identifying Sp1.2 as a positive regulator of hTERC transcription (compare figures 3B, re-analysed and re-plotted previous observations, and $3 \mathrm{C}$ ).

Thus, the role of Sp1.2 would appear to be dependent on the promoter context. Since both mutations have been shown to disrupt Sp1/Sp3 binding, the present results extend our view of the role of $\mathrm{Sp} 1.2$ in the core hTERC promoter, suggesting that Sp1.2 may act as both a positive and a negative regulatory site. This would be consistent with the ability of Sp1 sites to bind both positive or negative regulators and may also be consistent with a scenario in which co-operativity with downstream binding factors might recruit specific factors in a context dependent manner. In totality, the data confirm the role of Sp1.2 as a functional regulator of the hTERC promoter and provide the first evidence that a bona fide $h T E R C$ promoter mutation found in a human pathology may modulate core $h T E R C$ promoter activity in vitro.

\section{Discussion}

The hTERC mutation described in this study affects the core consensus sequence for one of $4 \mathrm{Sp} 1$ binding sites in the core hTERC promoter [13]. EMSA analysis indicated that the pathogenic mutation destroyed the binding of the transcriptional regulators Sp1 and Sp3 to this site (termed Sp1.2). Transient transfection of hTERC promoter-luciferase constructs confirmed that this site is a functional regulator of $h T E R C$ core promoter activity and that introduction of the patient mutation at this site modulates core hTERC promoter activity. Our data show that Sp1.2 has the capacity to mediate both positive and negative regulation of $h T E R C$, in a context dependent manner depending on the other sites available for transcription factor binding in the core promoter. This raises the possibility that Sp1.2 might exhibit functional co-operativity in vivo and that its role might vary, possibly even in a cell-specific manner, depending on the full complement of factors bound to the core promoter.

It is tempting to speculate that mutation of Sp1.2 might contribute to the pathogenesis of $\mathrm{PNH}$, especially given recent reports implicating perturbation of hTERC steady state levels in dyskeratosis congenita and in idiopathic aplastic anaemia [5-7]. The picture that is emerging is one in which the role of hTERC levels in the regulation of telomerase in non-malignant tissues may be more important than was previously thought. In support of this argument are the findings that haploinsufficiency of the telomerase RNA component is sufficient to result in shortened telomeres both in autosomal dominant dyskeratosis congenita in humans and in Terc $^{+/-}$heterozygous mice $[5,14]$. In Xlinked DC the mutations in DKC1 are thought to act by reducing the stability of hTERC leading to decreased telomerase levels [4], though they may also act through a defect in rRNA production [15]. Nevertheless it is interesting that amongst the DKC1 mutations is a point mutation in a conserved Sp1 binding site in the promoter [16]that in vitro has been shown to reduce transcription of the gene [17].

\section{Conclusions}

This is the first report of a $h T E R C$ mutation in a patient with PNH. This has important implications for the conventional model of PNH development. It is generally accepted that two factors are required for the development of PNH. One is bone marrow failure, in fact PNH clones are found in $~ 30 \%$ of AA patients and $~ 20 \%$ of myelodysplasia (MDS) patients. The second factor is a pathogenic PIGA mutation. The PNH clone is thought to expand because the GPI- cells have a growth or survival advantage over the GPI+ clones in the context of an aplastic bone marrow. The nature of the survival advantage is unknown but is thought to be mediated via one or more GPI linked proteins by an immunological mechanism. The implications of the present findings are that aplastic anaemia caused by a genetic mechanism can also lead to $\mathrm{PNH}$, suggesting the growth advantage of GPI- cells may be more subtle than resistance to cell killing via GPI linked proteins. It has been known for some time that cells from patients with aplastic anemia have significantly shorter telomeres than cells from matched controls [18-20]. This could be due to a direct effect on telomere synthesis or an indirect effect through for example increased cell death leading to increased proliferation of a smaller number of progenitor cells. Studies on $\mathrm{PNH}$ patients' telomere lengths have been inconsistent $[18,19]$ but a recent study of 10 patients with primary classical PNH showed granulocytes with significantly shorter telomeres than controls [21]. Moreover both GPI- and GPI+ cells showed telomere shortening suggesting it reflects the underlying aplastic anemia rather than the proliferation of the PNH clone. It 

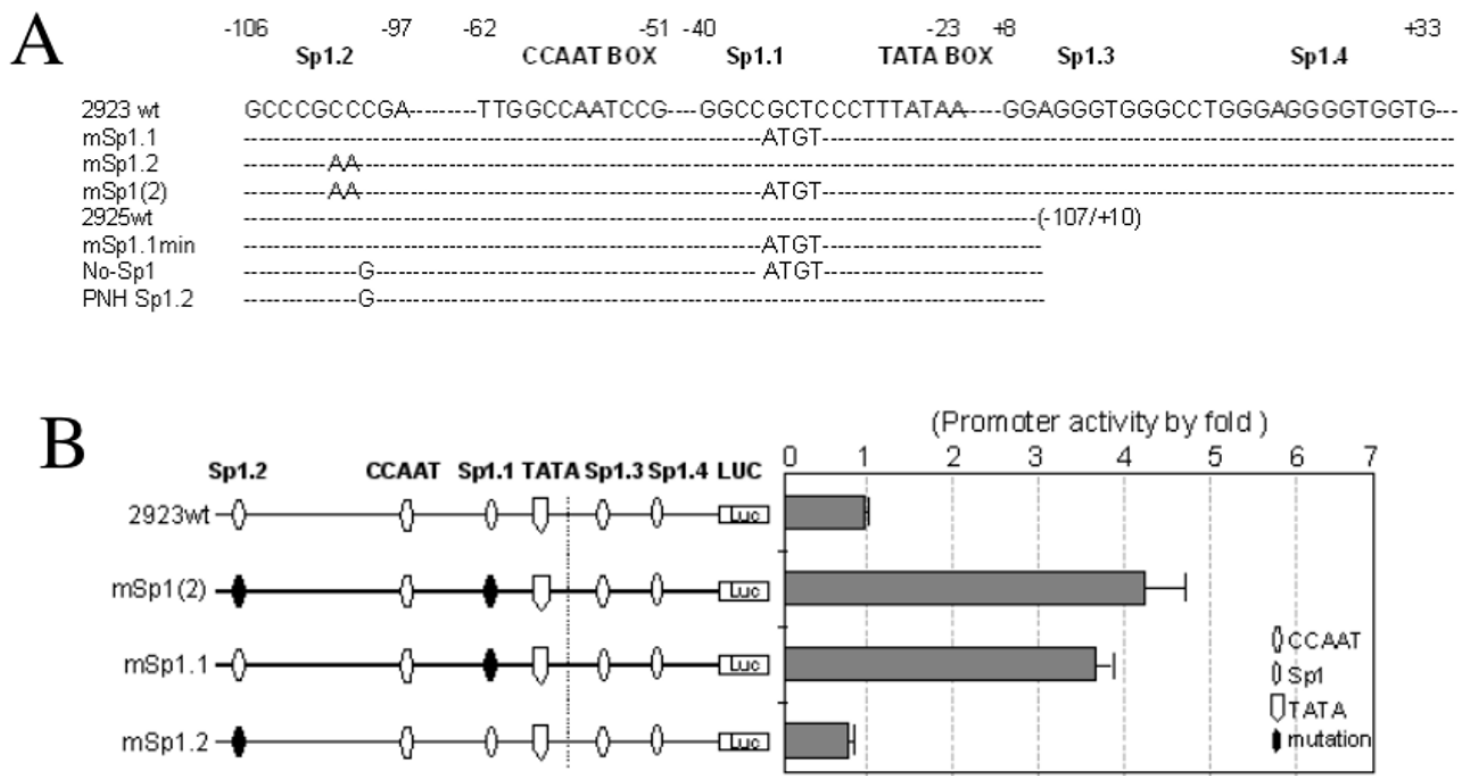

(Promoter activity by fold)

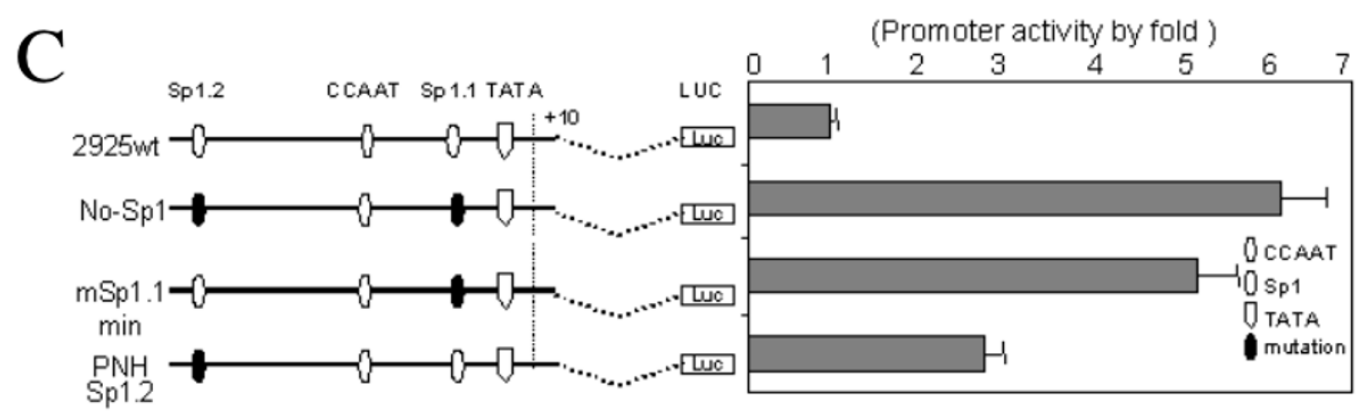

\section{Figure 3}

Transient transfection analysis A. The sequence of the wild-type hTERC core promoter (-107/+69) is shown at the top. The name of each mutant construct is indicated on the left hand side. The number on either side of the sequence is related to the transcriptional start site. Dashes indicate an identical sequence to wild-type. Mutated nucleotides are shown below the wild type sequence. B. Scanning mutational analysis Spl sites in the hTERC core promoter Promoter activities of site-replaced mutant constructs: The various symbols or circles represent the different transcription factor binding sites indicated at the top. Transcriptional start site indicated as broken line. The constructs are shown with a black ellipsoid shape indicating a sitereplaced mutation in one or more positions and open ellipsoids representing unmodified sites. The promoter activity is shown on the right hand side. Three micrograms of each plasmid were used for transient transfection analysis in 5637 cells. Promoter activities of the mutant constructs were assayed by transfection and compared to the wild-type promoter. The PRL-SV40 vector was used as an internal control to normalise the transfection efficiency. For each transfection the mean and standard deviation of data from three experiments are shown. C. Mutation of SpI.2 site from PNH patient in II 7 bp in length of minimum hTERC promoter increases promoter activity.

remains to be seen if $h T E R C$ mutations or other genetic disturbances of the telomere maintenance pathway are the exception or the rule in AA and PNH.

\section{Competing interests}

None declared.

\section{Authors' contribution}

WNK was responsible for the molecular analysis and wrote part of the paper. TV and AM performed the mutation analysis. $\mathrm{JZ}$ and $\mathrm{AB}$ did the binding and transfection assays. CA and BU examined the patient and provided the DNA. PJM instigated the collaboration and organized and 
contributed to the writing of the paper. $\mathrm{MB}$ provided a series of DNAs from PNH patients. NS and CE provided DNA from the ethnically matched controls. ID oversaw the clinical data analysis and wrote part of the paper. All authors contributed to the writing of the paper.

\section{Acknowledgements}

The authors would like to thank Aileen Monaghan for help in construct development. This work was supported in part by a Wellcome Trust Programme Grant.

\section{References}

I. Dokal I: Dyskeratosis congenita in all its forms. Br J Haematol 2000, I I 0:768-779.

2. Heiss NS, Knight SW, Vulliamy TJ, Klauck SM, Wiemann S, Mason PJ, Poustka A, Dokal I: X-linked dyskeratosis congenita is caused by mutations in a highly conserved gene with putative nucleolar functions. Nat Genet 1998, 19:32-38.

3. Filipowicz W, Pogacic V: Biogenesis of small nucleolar ribonucleoproteins. Curr Opinion Cell Biol 2002, I 4:319-327.

4. Mitchell JR, Wood E, Collins K: A telomerase component is defective in the human disease dyskeratosis congenita. Nature 1999, 402:55I-555.

5. Vulliamy T, Marrone A, Goldman F, Dearlove A, Bessler M, Mason PJ, Dokal I: The RNA component of telomerase is mutated in autosomal dominant dyskeratosis congenita. Nature 200I, 413:432-435.

6. Yamaguchi H, Baerlocher GM, Lansdorp PM, Chanock SJ, Nunez O, Sloand E, Young NS: Mutations of the human telomerase RNA gene (TERC) in aplastic anemia and myelodysplastic syndrome. Blood 2003, 102:916-918.

7. Vulliamy T, Marrone A, Dokal I, Mason PJ: Association between aplastic anaemia and mutations in telomerase RNA. Lancet 2002, 359:2168-2170.

8. Kinoshita $T$, Inoue N: Relationship between aplastic anemia and paroxysmal nocturnal hemoglobinuria. Int J Hematol 2002, 75: I17-122.

9. Bessler M, Schaefer A, Keller P: Paroxysmal nocturnal hemoglobinuria: insights from recent advances in molecular biology. Transfus Med Rev 200I, I5:255-267.

10. Zhao JQ, Hoare SF, McFarlane R, Muir S, Parkinson EK, Black DM, Keith WN: Cloning and characterization of human and mouse telomerase RNA gene promoter sequences. Oncogene 1998, I 6:1345-| 350 .

II. Zhao JQ, Glasspool RM, Hoare SF, Bilsland A, Szatmari I, Keith WN: Activation of telomerase rna gene promoter activity by NFY, SpI, and the retinoblastoma protein and repression by Sp3. Neoplasia 2000, 2:53I-539.

12. Yamada K, Tanaka T, Miyamoto K, Noguchi T: Sp family members and nuclear factor-Y cooperatively stimulate transcription from the rat pyruvate kinase $M$ gene distal promoter region via their direct interactions. B Biol Chem 2000, 275: |8|29-|8|37.

13. Zhao J, Bilsland A, Hoare SF, Keith WN: Involvement of NF-Y and SpI binding sequences in basal transcription of the human telomerase RNA gene. FEBS Lett 2003, 536: I II-119.

14. Hathcock KS, Hemann MT, Opperman KK, Strong MA, Greider CW, Hodes RJ: Haploinsufficiency of $m$ TR results in defects in telomere elongation. Proc Natl Acad Sci U S A 2002, 99:359I-3596.

15. Ruggero D, Grisendi S, Piazza F, Rego E, Mari F, Rao PH, CordonCardo C, Pandolfi PP: Dyskeratosis congenita and cancer in mice deficient in ribosomal RNA modification. Science 2003, 299:259-262.

16. Knight SW, Vulliamy TJ, Morgan B, Devriendt K, Mason PJ, Dokal I: Identification of novel DKCI mutations in patients with dyskeratosis congenita: implications for pathophysiology and diagnosis. Hum Genet 200I, I08:299-303.

17. Salowsky R, Heiss NS, Benner A, Wittig R, Poustka A: Basal transcription activity of the dyskeratosis congenita gene is mediated by SpI and Sp3 and a patient mutation in a Sp I binding site is associated with decreased promoter activity. Gene 2002, 293:9-19.
18. Brummendorf TH, Maciejewski JP, Mak J, Young NS, Lansdorp PM: Telomere length in leukocyte subpopulations of patients with aplastic anemia. Blood 200I, 97:895-900.

19. Ball SE, Gibson FM, Rizzo S, Tooze JA, Marsh JC, Gordon-Smith EC: Progressive telomere shortening in aplastic anemia. Blood 1998, 91 1:3582-3592.

20. Lee JJ, Kook H, Chung IJ, Na JA, Park MR, Hwang TJ, Kwak JY, Sohn SK, Kim HJ: Telomere length changes in patients with aplastic anaemia. Br J Haematol 200I, I I 2: I025-1030.

21. Karadimitris A, Araten DJ, Luzzatto L, Notaro R: Severe telomere shortening in patients with paroxysmal nocturnal hemoglobinuria affects both GPI- and GPI+ hematopoiesis. Blood 2003 , 102:5|4-5|6.

\section{Pre-publication history}

The pre-publication history for this paper can be accessed here:

http://www.biomedcentral.com/1471-2326/4/3/prepub
Publish with Bio Med Central and every scientist can read your work free of charge

"BioMed Central will be the most significant development for disseminating the results of biomedical research in our lifetime. " Sir Paul Nurse, Cancer Research UK

Your research papers will be:

- available free of charge to the entire biomedical community

- peer reviewed and published immediately upon acceptance

- cited in PubMed and archived on PubMed Central

- yours - you keep the copyright

Submit your manuscript here:

http://www.biomedcentral.com/info/publishing_adv.asp
BiolMedcentral 